Revue internationale P.M.E.

Économie et gestion de la petite et moyenne entreprise

\title{
Une analyse du processus de structuration des industries du capital-risque
}

\section{Patrice Geoffron}

Volume 4, numéro 3, 1991

URI : https://id.erudit.org/iderudit/1008072ar

DOI : https://doi.org/10.7202/1008072ar

Aller au sommaire du numéro

Éditeur(s)

Presses de l’Université du Québec

ISSN

0776-5436 (imprimé)

1918-9699 (numérique)

Découvrir la revue

Citer cette note

Geoffron, P. (1991). Une analyse du processus de structuration des industries du capital-risque. Revue internationale P.M.E., 4(3), 95-114.

https://doi.org/10.7202/1008072ar

\section{Résumé de l'article}

Le capital-risque est une innovation dïntermédiation dédiée au financement des PME. L'article propose une analyse de la formation de cette innovation. Cette analyse est fondée sur une grille qui articule les deux schémas dominants (et complémentaires) des théories de l'innovation financière : la théorie de l'innovation financière induite par la « demande de nouvelles caractéristiques » et de l'innovation financière induite par les « contraintes des institutions». Ces schémas permettent, respectivement, de mettre en exergue la nouvelle fonction productive des PME (et les besoins qui en résultent) et l'action d'institutions (publiques, grandes entreprises et banques) comme déterminants principaux de l'innovation « capital-risque ». La pertinence de ces relations est ensuite testée sur deux modèles de formation du capital-risque : il apparaît que le modèle américain, matrice du capital-risque, s'inspire d'une induction par la demande de caractéristiques de l'innovation privée, tandis que le modèle français se base sur une induction par les contraintes des institutions selon une logique d'innovation publique.
Ce document est protégé par la loi sur le droit d'auteur. L'utilisation des services d’Érudit (y compris la reproduction) est assujettie à sa politique d'utilisation que vous pouvez consulter en ligne.

https://apropos.erudit.org/fr/usagers/politique-dutilisation/ 


\title{
Une analyse du processus de structuration des industries du capital-risque
}

\author{
Patrice GEOFFRON \\ Université Paris XIII*
}

\begin{abstract}
RÉSUMÉ
Le capital-risque est une innovation d'intermédiation dédiée au financement des PME. L'article propose une analyse de la formation de cette innovation. Cette analyse est fondée sur une grille qui articule les deux schémas dominants (et complémentaires) des théories de l'innovation financière: la théorie de l'innovation financière induite par la " demande de nouvelles caractéristiques " et de l'innovation financière induite par les "contraintes des institutions ". Ces schémas permettent, respectivement, de mettre en exergue la nouvelle fonction productive des PME (et les besoins qui en résultent) et l'action d'institutions (publiques, grandes entreprises et banques) comme déterminants principaux de l'innovation "capital-risque ". La pertinence de ces relations est ensuite testée sur deux modèles de formation du capital-risque : il apparaît que le modèle américain, matrice du capital-risque, s'inspire d'une induction par la demande de caractéristiques de l'innovation privée, tandis que le modèle français se base sur une induction par les contraintes des institutions selon une logique d'innovation publique.
\end{abstract}

\begin{abstract}
Venture capital is a financial innovation. Such an instrument reduces the eviction of innovative small business from both banking and financial markets. This paper is concerned with venture capital formation. Our analysis is based on financial innovation two main theoretical schemes: "financial innovation induced by demand of new characteristics" theory and "financial innovation induced by constraints of institutions " theory. The first scheme underlines small business new industrial functions (and new needs) and the second underlines institutional strategies (governements, firms and banks) as causes of venture capital formation. The relevance of this theoretical framework is then tested on two models of venture capital formation: the US model, matrix of venture capital, seems to be related to a "new demand" inducement and the french model to a "constraints of institutions " inducement.
\end{abstract}

* Patrice Geoffron est Maître de Conférences à l'Université de Paris XIII et membre du Centre de recherche en économie industrielle (CREI). Il est spécialiste des questions relatives au financement des PME et aux mutations des industries financières. 


\section{RESUMEN}

El capital riesgo es una innovación creada para la financiación de las PYMES. El artículo propone hacer un análisis del desarrollo de esta innovación. Análisis que se basa sobre un sistema que articula los dos esquemas dominantes (y complementarios) de las teorias de la innovación financiera: la inducila por "la demanda de nuevas caracteristicas" y la inducida por "la demanda de nuevas caracteristicas" y la inducida por "las obligaciones de las instituciones". Cada uno de estos esquemas permite poner de relieve la nueva función productiva de las PYMES (y las necesidades que se deriven) y la acción de las instituciones (públicas, grandes empresas y banca) como determinantes principales des capital riesgo. Se ha comprobado la conveniencia de interrelacionar las dos variables a través de dos modelos de formación de capital riesgo: el americano y el francés. De su análisis se deduce que el modelo americano se inspira en la lógica de la demanda de las caracteristicas de la innovación privida, mientras que el modelo francés se basa en las obligaciones de las instituciones públicas.

\section{Introduction}

Innovation financière instituée aux États-Unis, puis diffusée progressivement en Europe aux cours des deux dernières décennies, le capital-risque est un instrument de développement, principalement développé pour les PME lors d'une phase de croissance, qui combine un apport de fonds propres et de compétences (techniques, stratégiques ou financières). Il constitue une possibilité de financement pour des PME innovatrices qui ne peuvent avoir accès aux marchés de capitaux et aux financements bancaires en raison du degré de risque que présentent en général leurs investissements. Des industries financières nouvelles se sont structurées autour du produit capital-risque : en 1989, elles géraient 33 milliards de dollars de ressources globales aux États-Unis et 27 milliards de dollars en Europe ${ }^{1}$.

Aussi, nous présentons ici :

- Une analyse des conditions de formation de cette innovation financiere en nous efforçant, tout particulièrement, de révéler les modalités de structuration des industries financieres du capital-risque et de différencier l'expérience américaine de l'expérience française en la matière.

1. Statistiques de Venture Economics pour les États-Unis et de l'European Venture Capital Association (EVCA) pour l'Europe. Par ailleurs, on recourra également aux statistiques de l'Association française des investisseurs en capital-risque (AFIC).

2. Cette contribution reprend les principaux résultats d'une thèse de doctorat intitulée : "La formation d'une innovation financière : le capital-risque ». Une analyse comparative des modèles américain et français, Université Paris XIII, 1990. Cette thèse a obtenu le Prix 1991 de l'Association nationale des docteurs es sciences-économiques. 
- Dans cette perspective, nous proposerons une grille d'analyse appuyée sur les théories de l'innovation financiere, en nous référant principalement aux schémas de l'induction par la demande de nouvelles caractéristiques et par les contraintes des institutions ${ }^{2}$.

Notre propos ne vise pas l'apport d'éléments nouveaux dans la compréhension des mécanismes d'investissement. Ce type d'analyse micro-économique constitue, en effet, une part majeure de la littérature consacrée au capital-risque. Notre perspective est plutôt de nature méso-économique, puisque nous relèverons les facteurs à l'origine de la création, puis de l'essor des industries du capital-risque, pour dessiner ensuite les trajectoires française et américaine typiques.

\section{Les spécificités d'une innovation d'intermédiation}

\subsection{Le recours à l'intermédiation pour la gestion du financement à risque}

Le capital-risque représente une issue à l'impasse où aboutit le financement des PME innovatrices, tout particulièrement pour les plus jeunes encore au stade de création. La taille de ces entreprises est insuffisante pour avoir accès aux marchés de capitaux, tandis que leurs besoins excèdent généralement leurs capacités d'autofinancement. En outre, les PME présentent des risques qui entravent un recours massif aux emprunts bancaires et des caractéristiques qui les rendent particulièrement vulnérables aux stratégies de sélection adverse ${ }^{3}$ développées par les banques (Heggestad et Shepherd, 1982).

Le financement de ces entreprises, caractérisé par une information imparfaite et asymétrique, impose ainsi le recours à des intermédiaires financiers spécialisés. Les organismes de capital-risque contribuent à optimiser la gestion

3. Conformément à la théorie de l'agence, les banques constituent un groupe dominant. Cette position les autorise à faire une sélection de façon à exclure les agents emprunteurs les moins sûrs au regard du risque de défaut qu'ils présentent. Toutefois, au lieu de procéder à une sélection conventionnelle par les prix en augmentant le taux d'intérêt, les banques ont avantage à opérer un rationnement par les quantités. En effet, dans la première hypothèse, les banques peuvent certes espérer un accroissement de leurs revenus, toutes choses étant égales par ailleurs ; mais les entrepreneurs seront incités à préférer les projets d'investissement les plus rémunérateurs, mais également les plus risqués. À l'inverse, les entrepreneurs qui manifestent une forte aversion à l'égard du risque tendent à sortir du marché des fonds prêtables. In fine, le bailleur de fonds enregistre donc une élévation du degré de risque qu'il encourt. Dans la seconde alternative de rationnement par les quantités, les banques peuvent fixer un prix en deçà de l'équilibre, mais exclure les emprunteurs les moins fiables. 
de cette information en centralisant les analyses, induisant ainsi une optimisation des coûts de transaction et assurant une divulgation restreinte. Y. Chan (1983) a ainsi démontré que grâce à ces intermédiaires spécialisés dans le capital-risque les entrepreneurs lanceront des projets présentant une rentabilité attendue et un risque plus élevé. Dotés d'une meilleure information que le reste du marché, ces intermédiaires seront aptes à diversifier leur portefeuille afin d'en réduire le risque global.

\subsection{Le cycle de production des Industrles du capital-risque}

Le capital-risque est donc un processus d'intermédiation novateur. La rémunération des fonds engagés par les organismes de capital-risque (OCR) procède essentiellement de plus-values de cession et n'est ni prédéterminée ni plafonnée, engendrant souvent une rentabilité de portefeuilles très concurrentielle du point de vue des offreurs de capitaux.

Le processus de production de l'industrie du capital-risque comprend six phases.

1. La collecte des capitaux par les OCR auprès de banques, caisses de retraite, compagnies d'assurance, grandes entreprises ou investisseurs individuels.

2. La confrontation des OCR et des demandeurs de capitaux (prospection, propositions spontanées, prescripteurs intermédiaires)...

3. ... parmi lesquels s'effectue une sélection rigoureuse qui conduira généralement à ne retenir que de 5 à $10 \%$ des dossiers.

4. Un contrat est élaboré qui définit la nature et le volume des apports de capitaux, ainsi que les relations qu'entretiendront l'entreprise et l'OCR à l'issue du transfert de fonds.

5. En effet, ce transfert n'interrompt pas les relations ; l'OCR est désormais actionnaire de l'entreprise et accompagne, et parfois même dicte son évolution par un apport de compétences ${ }^{4}$.

6. Enfin, au terme d'un engagement de 5 à 10 ans (en moyenne), l'OCR cédera sa participation lors d'une introduction de l'entreprise sur un Second Marché boursier ou bien encore auprès d'une entreprise industrielle ou d'une autre institution financière.

4. Détermination des options stratégiques, résolution de problèmes juridiques, interface entre l'entreprise et un réseau des relations industrielles ou financières, voire participation très active à la gestion qualifiée de stratégie "hands in" (par opposition à une stratégie " hands off " plus distante). 
Toutes les opérations de capital-risque sont effectuées suivant ce cycle, mais ne présentent pas de contraintes ou de risques homogènes. Le marché du capital-risque est en fait constitué de deux segments principaux. On distingue le capital-création, qui regroupe les opérations de financement des entreprises en phase de constitution et de structuration ${ }^{5}$, du capital-développement, qui s'adresse aux entreprises plus mûres en phase d'expansion. Sur le premier segment, le risque d'investissement est aigu pour les OCR en raison des hypothèques techniques et commerciales qui pèsent sur les entreprises en création, comme l'indique leur taux de mortalité élevé. Sur le segment du capital-développement, les entreprises sortent de leur période d'adolescence et occupent une part de marché significative. Si les structures de l'entreprise sont moins précaires, le capital-développement correspond généralement à des engagements financiers très supérieurs au capital-création, d'où des échecs individuellement plus pénalisant pour les $\mathrm{OCR}^{6}$.

L'art des OCR, dans le cadre du cycle de production qui vient d'être décomposé, consiste largement à analyser et gérer des risques. Le financement par fonds propres place directement les OCR dans la position du banquier schumpeterien, en charge du risque de l'entreprise (Schumpeter, 1983). Ces risques sont protéiformes : perte ou immobilisation durable des capitaux investis, mobilisation excessive des capacités d'analyse au détriment des autres entreprises en portefeuille, responsabilisation juridique de l'OCR en cas de faillite, etc. Le niveau global de risque d'un investissement apparaît en moyenne comme négativement corrélé à l'âge de l'entreprise et positivement au degré d'innovation de son activité.

\subsection{Une Innovation financière hybride et spécifique}

L'apparition de l'innovation financière capital-risque est inscrite dans une vaste mutation de la sphère financière qui se manifeste avec une acuité particulière depuis le début de la décennie 80 . Cette mutation combine l'influence des innovations « spontanées » des agents financiers (et dans une moindre mesure non financiers) et d'autres qui résultent, directement ou indirectement, d'une modi-

5. Nous englobons dans cette catégorie le « capital de départ » qui est relié aux phases les plus en amont de l'élaboration du projet d'entreprise.

6. Précisons également qu'un troisième segment du marché du capital-risque s'adresse aux entreprises en phase de cession. Ce capital-transmission ne procède cependant pas des mêmes mécanismes que le capital-risque stricto sensu, mais il constitue un marché de diversification important pour les OCR. Sahlman $(1990: 518)$ indique de manière plus générale que la méthode d'organisation du capital-risque est transférable à d'autres types d'opérations de financement. 
fication des stratégies réglementaires des pouvoirs publics. La dynamique innovatrice trouve à sa source des facteurs internes à la sphère financière (contraintes macrofinancières, données concurrentielles et réglementaires, etc.) ou répond à des évolutions de l'économie réelle selon deux axes : une intégration des progrès technologiques, une prise en compte des signaux apparentés à de nouveaux besoins.

Au sein de cette mutation globale, l'innovation capital-risque se distingue par un caractère hybride et spécifique.

L'hybridisme est manifeste à deux niveaux. En premier lieu, le capitalrisque n'est pas réductible à un simple produit financier, puisqu'il incorpore un apport de services. En second lieu, un recours à la typologie schumpeterienne des innovations (Schumpeter, 1983) nous révèle que le capital-risque est à la fois :

- une innovation de produit par une combinaison originale de capitaux propres et de compétences ;

- une innovation de processus, car l'intermédiation à risque obéit aux mécanismes originaux qui ont été décrits et qui la différencient notamment de l'intermédiation bancaire ;

- une innovation d'organisation, car une industrie financière nouvelle s'est structurée autour du produit capital-risque.

La spécificité est également double. En premier lieu, nous l'avons indiqué, le capital-risque est une innovation d'intermédiation, alors que les évolutions financières majeures des années 80 relèvent plutôt d'innovations de mise en marché ? L'explication tient à la nature des entreprises financées, pour lesquelles les financements par le marché ne sont pas optimaux 7 . Le capital-risque paraît ainsi occuper une niche financière, un interstice du marché financier. En second lieu, les tendances lourdes des mutations n'ont pas été favorables au financement de l'innovation et aux engagements à long terme (Aglietta, 1987), alors que précisément le capital-risque correspond à un investissement de longue durée dans des entreprises innovatrices.

7. En raison notamment des asymétries informationnelles entre offreurs et demandeurs de capitaux que comporteraient des opérations de financement par le marché de ce type d'entreprises. 


\section{La grille d'analyse de la formation de l'innovation capital-risque}

Aussi bien les révolutions marchandes de la Renaissance que les révolutions industrielles du $\mathrm{XIX}^{\mathrm{e}}$ siècle et du début du $\mathrm{xx}^{\mathrm{e}}$ ont requis des formes de financement à risque plus sophistiquées. L'innovation capital-risque composant le dernier stade de ce processus multiséculaire, nous nous arrêterons maintenant à l'analyse des déterminants de cette innovation financière.

\subsection{La configuration des théories de l'innovation financière}

Les innovations financières n'ont longtemps suscité qu'une littérature peu abondante. La réglementation née de la crise de années 30 qui a prévalu pendant plus de 40 ans, a sans aucun doute inhibé les mutations financières et focalisé les intérêts sur les innovations réelles comme facteur de croissance économique. Aussi, le concept d'innovation financière n'est-il introduit qu'au cours de la première moitié des années 70 par S. Greenbaum, C. Haywood (1971) et W. Silber (1975).

Nous proposons une grille de lecture des déterminants de l'innovation capital-risque dont l'axe majeur articule les deux principaux schémas théoriques de la causalité des innovations financières :

- l'innovation induite par la demande de nouvelles caractéristiques;

- l'innovation induite par les contraintes des institutions.

Le premier schéma explicatif majeur est dérivé de la théorie lancasterienne de la consommation (Lancaster, 1966). Chaque instrument monétaire ou financier peut en effet, comme l'a avancé J. Tobin, être réduit à une dizaine de caractéristiques, dont : la liquidité, la divisibilité, la réversibilité-substituabilité, le rendement, etc. (Greenbaum et Haywood, 1971). L'ensemble des combinaisons de ces caractéristiques conduit à distinguer deux catégories: les instruments disponibles et les instruments virtuels. Dans ce cadre, l'innovation financière résulte d'une demande par les agents d'instruments monétaires ou financiers dotés de caractéristiques nouvelles et s'interprète comme le transfert d'un instrument de la catégorie des virtuels vers la catégorie des disponibles (Hardouin, 1973).

La théorie de l'innovation induite par les contraintes des institutions a été proposée par W. Silber (1975). En substance, l'innovation financière s'interprète comme la réponse d'organisations (intermédiaires financiers, pouvoirs publics, grandes entreprises) aux évolutions de leurs contraintes endogènes (ratio de liquidité, de solvabilité, objectif de croissance, objectif de rentabilité, etc.) et 
exogènes (liées à l'état de la concurrence ou à la réglementation en vigueur). Chaque organisation doit optimiser sa fonction-objectif au sein de ce système de contraintes. Chacune des contraintes est couplée à une variable duale qui représente la variation d'utilité relative à un desserrement marginal de la contrainte. Un prix fictif correspondant au coût du «non-contournement de la contrainte " ou " coût d'adhésion » en est déduit (Silber et Ben Horim, 1977). On touche ici au cour du raisonnement de W. Silber: l'acuité de l'incitation à innover est proportionnelle à l'accroissement de ce coût d'adhésion. L'adaptation innovatrice n'est cependant pas instantanée. Elle suppose tout d'abord que le nouveau signal soit persistant et perçu comme durable par l'organisation. $\grave{A}$ ce décalage initial s'ajoutent des délais d'adaptation de l'innovation. Le coût d'adoption de l'innovation doit évidemment rester inférieur à celui de l'adhésion à la contrainte. Les conséquences de la réglementation $Q$ aux États-Unis, par exemple, s'insèrent parfaitement dans le cadre d'analyse de W. Silber ${ }^{8}$.

Ces deux schémas pourraient a priori sembler antinomiques, puisque le premier impute préférentiellement l'innovation financière à des facteurs de demande, tandis que le second met en exergue les facteurs d'offre. En fait, ils sont largement complémentaires car, tout comme l'ont souligne D. Mowery et $\mathbf{N}$. Rosenberg (1979) dans le domaine réel, de nombreuses innovations financières relèvent d'une interaction de facteurs multiples et conjoints, sans prédominance manifeste de facteurs d'offre ou de demande.

L'innovation capital-risque s'apparente à ce type d'agencement complexe des facteurs explicatifs. Aussi pour analyser la formation du capital-risque, proposons-nous successivement une lecture du phénomène dans la perspective d'une induction par la demande de nouvelles caractéristiques et d'une induction par les contraintes des institutions.

\subsection{La lecture du phénomène selon le schéma d'induction de l'Innovation par la demande de nouvelles caractéristiques}

Une lecture opérée selon le premier schéma d'induction conduit à mettre tout d'abord en exergue le renouveau de l'innovation comme attribut de la fonction d'entrepreneur. Après que la préminence des grandes entreprises a fait croire à la disparition des petites entreprises, la création accélérée de ces dernières depuis plus de 10 ans a réaffirmé l'importance des entrepreneurs et son rôle de catalyseur de l'innovation. Plus généralement, la période récente a révélé les qualités des PME pour traverser les crises et leur importance tant au plan de l'emploi et du développement local, que de l'innovation. Dans ce dernier domaine, si la capacité des PME a longtemps nourri un débat (Acs et Audretsch, 1988), il est aujourd'hui admis que leur productivité est souvent supérieure à

8. Réglementation de la rémunération des dépôts à vue.

Revue internationale P.M.E., vol. 4, n 3, 1991 
celle des grandes entreprises dans ce domaine. Tout particulièrement, l'essor de secteurs nouveaux à la fin des années 70 a permis à des petites entreprises de technologie avancée de donner la mesure de cette capacité en matière innovatrice.

Dans ce contexte, la naissance du capital-risque, si l'on retient la logique du schéma de l'innovation financière induite par la demande de nouvelles caractéristiques, s'interprète donc tout d'abord comme la conjugaison de besoins de fonds propres et de compétences externes exprimés ou latents chez les PME. Ces besoins sont particulièrement marqués chez les entreprises jeunes ou au développement accéléré et bien sûr, chez les PME innovatrices, potentiellement soumises à des turbulences importantes. Le composé « fonds propres + compétences » constitue en substance un outil de gestion des stades ou des contenus d'activité qui présentent un risque aigu.

Le transfert du capital-risque du sous-ensemble des instruments virtuels à celui des instruments disponibles apporte une solution originale aux entreprises dont les caractéristiques financières limitent un recours à des fonds immédiatement onéreux (crédits à MLT) et à des prestations de conseils tarifées.

\subsection{La lecture du phénomène selon le schéma d'induction de l'innovation par les contraintes des institutions}

Une lecture selon le schéma des contraintes des institutions conduit essentiellement à considérer l'action des pouvoirs publics, des grandes entreprises et des banques pour la formation du capital-risque.

Les contraintes nouvelles enregistrées par les Pouvoirs Publics au cours des années 70 sont liées à leurs responsabilités dans la gestion des mutations industrielles et technologiques, et notamment, leurs traductions en termes d'emploi et de développement local. Alors même que la croissance fordiste appuyée sur les grandes unités marquait ses limites, ce faisceau de contraintes nouvelles a conduit les pouvoirs publics à mieux tenir compte des PME dans le cadre des politiques industrielles (Rainelli, 1986). Ont ainsi été élaborées des politiques destinées à protéger ces entreprises dans un environnement adverse ou à encourager leurs qualités originales (notamment en matière d'innovation). La promotion du capital-risque par les pouvoirs publics est apparentée à cette logique9. Trois types de mesures ont été mis en œuvre:

- des mesures institutionnelles par une modification de l'approche du risque et du champ d'intervention des institutions financières publiques ou par une conception de structures juridiques d'offre de capital-risque ;

9. Même s'il faut rappeler que l'expérience des Sociétés de développement régional remonte en France aux années 50. 
- des mesures réglementaires destinées à assouplir l'accès des marchés des capitaux ou à faciliter l'offre de fonds des investisseurs institutionnels ;

- des mesures fiscales enfin, soit par une déduction des pertes sur investissement, soit par une taxation avantageuse des gains (OCDE 1986).

Au cours des années 70, des contraintes nouvelles de concurrence et de mutation se sont également imposées aux grandes entreprises. Comme la technologie occupe désormais une pondération supérieure dans la détermination des stratégies de firme, les grandes entreprises ont, comme les pouvoirs publics, été sensibles à la démonstration faite par certaines PME de leurs capacités exploratoires en matière technologique. Elles ont dès lors été intéressées à des formes de partenariat nouvelles avec des PME, afin d'exercer une veille technologique. C'est pourquoi les grandes entreprises ont encouragé le développement du capital-risque à trois niveaux :

- en contribuant tout d'abord à l'offre de fonds aux OCR ;

- en constituant directement des filiales financieres spécialisées dans le capital-risque ;

- en prenant le relais des OCR dans les phases de désengagement.

Les banques, enfin, ont subi une concurrence accrue des marchés financiers sous l'influence des innovations et de la déréglementation financières. Bien qu'elles aient répondu à la désintermédiation en concevant une nouvelle intermédiation de marché, leurs activités traditionnelles ont été largement affectées. Dès lors, le marché des PME a présenté un intérêt nouveau et a requis l'élaboration de produits et de services adaptés, d'où l'attrait du capital-risque comme segment de diversification. Bien que les banques aient souvent été accusées de dénaturer l'activité de capital-risque en l'intégrant à des modes de raisonnement propres à l'activité de prêt, de facto elles occupent dans certains pays une part du marché très importante (en France, notamment) et pèsent sur les trajectoires des industries du capital-risque.

\section{La différenciation des modèles américain et français}

Le développement du capital-risque aux États-Unis et en France s'est effectué selon des modalités sensiblement différentes que nous soulignerons en recourant à notre grille d'analyse enrichie par la distinction faite par $\mathrm{C}$. de Boissieu (1986) entre les processus nationaux privés ou publics d'innovation financière. 


\subsection{Des trajectoires de développement du capital-risque...}

Le capital-risque moderne a été institué aux États-Unis après la Seconde Guerre mondiale, tout d'abord sous l'impulsion de détenteurs de fortunes privées, relayés à partir de la fin des années 50 par les pouvoirs publics qui créent les Small Business Investment Companies (SBICs). Néanmoins, l'industrie du capital-risque restera longtemps embryonnaire et les niveaux de collecte et d'investissement seront même pratiquement annulés au milieu des années 70 . Ce ne sera qu'à partir de 1978 qu'interviendra le démarrage de l'industrie du capital-risque sous l'effet :

- d'une amélioration du cadre réglementaire et fiscal et

- de l'apparition de nombreuses occasions d'investissement au sein des secteurs technologiques nouveaux.

Dès lors, les investissements et la collecte de capitaux s'accroîtront à un rythme exponentiel, notamment grâce aux apports massifs de capitaux des investisseurs institutionnels. Pourtant, en 1984 et 1985, cet afflux non régulé de capitaux conduira à une crise provoquée par une surcapitalisation des entreprises financées et une saturation du marché boursier de désengagement. Si les indicateurs d'activité ont retrouvé des niveaux très élevés en 1986 et $1987^{10}$, l'industrie semble intrinsèquement soumise à des évolutions erratiques fréquentes, comme en atteste un nouveau recul des investissements et de la collecte de capitaux en 1988. On retiendra néanmoins que le volume du portefeuille global (indicateur de stock et non de flux) s'est toujours accru de façon régulière en dépit de ces retournements conjoncturels, pour s'établir à plus de 30 milliards de dollars en 1989 (voir graphiques en annexes).

La structuration d'une industrie du capital-risque en France est à placer dans la perspective du développement d'une industrie européenne qui fut impulsé par la constitution de fonds privés ou institutionnels multinationaux dans les années 70, puis accompagné par une série d'actions de la Commission européenne (études de marché, aides à la constitution d'OCR). Aujourd'hui, le marché européen reste cependant caractérisé par une hétérogénéité des marchés nationaux et une forte concentration des capacités d'investissement : la somme des trois premiers pays (Grande-Bretagne, France, Pays-Bas) représentait ainsi en 1989 respectivement $85 \%$ de la collecte de capitaux, $81 \%$ des investissements et $80 \%$ du portefeuille global.

L'adaptation d'une industrie du capital-risque en France a eu lieu sous l'égide des pouvoirs publics qui ont défini les cadres juridiques des OCR (Société de financement de l'innovation (1972), Fonds communs de placement

10. Plus de 4,5 milliards de dollars ont été collectés au cours de ces deux années.

Revue internationale P.M.E., vol. 4, $n^{\circ}$ 3, 1991 
à risque (1983), Sociétés de capital-risque (1985), etc.) tout en s'efforçant de favoriser les désengagements (Second marché) ou le partage des risques (Sofaris $\left.{ }^{11}\right)$. Cette politique n'a porté ses fruits qu'à partir du début des années 80, soit après de longs ajustements. Aujourd'hui, le marché français se situe au second rang européen, derrière la Grande-Bretagne, avec un volume global d'investissements de 4700 millions de francs ${ }^{12}$. L'apport important de capitaux bancaires a notamment permis, à partir de 1983 , une rapide montée en puissance de l'activité.

\section{2. ...quil reflètent des processus d'innovation financière hétérogènes}

Soumettons maintenant ces processus à la grille de lecture présentée à la section précédente.

Les formations de l'innovation "capital-risque » aux États-Unis et en France sont affiliées à des " traditions" de transformations des sphères financières très différentes. Si certains déterminants macro-économiques sont communs (évolution du contexte inflationniste, volatilité des taux d'intérêts et des taux de change, etc.), les schémas adoptés divergent largement :

- aux États-Unis, les modalités sont décentralisées et privées; elles concourent aux enchaînements d'une dialectique réglementaire : réglementation par les autorités $\rightarrow$ contournement par les agents des dispositions réglementaires $\rightarrow$ déréglementation ou re-réglementation (Kane, 1983);

- en France, elles sont centralisées et publiques, tandis que le degré élevé de réglementation est un facteur d'inhibition des innovations privées (de Boissieu, 1986).

Les processus de l'innovation «capital-risque » présentent des dissymétries aussi marquées qui s'apparentent en partie aux précédentes.

Dans le cas américain, la demande de nouvelles caractéristiques, exprimée par les petites entreprises des nouveaux secteurs (électronique, informatique et biotechnologie principalement), a été le principal déterminant à l'origine de l'innovation. Elle a engendré une dynamique largement privée et indépendante de stratégies institutionnelles. L'influence des institutions sur le processus innovateur s'est toutefois manifestée en réponse à des contraintes de développement local (États régionaux) ou de gestion technologique (grandes entreprises), mais elle est restée marginale. Certes, les investisseurs

11. Société française de l'assurance du capital-risque.

12. L'industrie française du capital-risque détenait, en $1989,19 \%$ du portefeuille européen. 
institutionnels (caisses de retraite et assurances) contribuent aujourd'hui largement à l'offre de capitaux aux OCR, mais ils ont accompagné la croissance de l'industrie sans l'inciter. Même s'ils sont désormais des acteurs primordiaux de la sphère du capital-risque, leur stratégie s'apparente plus à celle de suiveurs que d'innovateurs.

Le pouvoir central, selon une logique libérale, n'est intervenu directement que sur des segments connexes au capital-risque stricto sensu et non couverts par des agents privés. Ces mesures générales réglementaires et fiscales ont influencé la trajectoire de l'industrie du capital-risque (négativement ou positivement), mais toujours de façon indirecte et non dans le cadre d'une politique volontariste comme en France.

$\mathrm{Au}$ total, l'induction par la demande de nouvelles caractéristiques prédomine dans le modele américain selon une logique d'innovation privée.

La configuration française diffère profondément du cas américain, lequel servit pourtant de référence. En effet, la formation du capital-risque en France doit tout d'abord s'interpréter comme une diffusion du modèle américain qui inspira la création des premiers OCR au début de la décennie 70. Mais, la spécificité marquée des modalités retenues fait toutefois de ce processus, sinon un processus d'innovation original, du moins un processus de diffusion innovant.

Alors que le schéma américain s'apparente principalement à une relation du type "demande $\rightarrow$ offre ", le schéma français est affilié à la relation inverse. Les pouvoirs publics se sont efforcés de structurer une offre afin de promouvoir le développement des PME innovatrices, à partir, d'un tissu industriel manifestement moins apte qu'aux États-Unis à susciter l'émergence spontanée d'entreprises de haute technologie dès le départ.

Nous l'avons indiqué, les pouvoirs publics ont contribué à la délimitation de tout le cadre fiscal et réglementaire, et à la création d'institutions complémentaires aux intermédiaires à risque. La structuration d'une industrie du capital-risque en France a pourtant requis plusieurs itérations en raison d'un cadre économique et financier trop éloigné de la référence américaine pour une simple transposition des mécanismes en œuvre outre-Atlantique. L'entrée massive des banques au sein de l'industrie du capital-risque à partir du début de la décennie 80 a également contribué à son décollage et à l'évolution des pratiques. Le processus français a pour moteurs les stratégies des institutions publiques et bancaires, considérant respectivement le capital-risque dans la perspective de leurs contraintes de politique industrielle et de politique concurrentielle.

$\mathrm{Au}$ total, l'induction par les contraintes des institutions prédomine dans le modele français selon une logique d'innovation publique. 
Les activités de capital-risque aux États-Unis et en France different tout comme les processus innovateurs qui les ont engendrées. Les logiques directrices sont certes les mêmes et consistent à valoriser des capitaux propres par un engagement à moyen ou long terme dans des PME. Mais dans le cadre de ces principes génériques, les acteurs et les pratiques sont distincts.

\section{Caractéristiques dominantes des processus d'innovation capital-risque aux États-Unis et en France}

\begin{tabular}{|c|c|c|c|}
\hline & & Etats-Unis & France \\
\hline $1^{\circ} \mathrm{I}$ & $\begin{array}{l}\text { Demande de nouvelles } \\
\text { caractéristiques }\end{array}$ & $\begin{array}{l}\text { Majeure } \\
\hat{A} \text { tous les stades du } \\
\text { processus d'innovation }\end{array}$ & $\begin{array}{l}\text { Modérée } \\
\text { Réduction progressive de } \\
\text { l'inadéquation offre/demande }\end{array}$ \\
\hline $2^{\circ} \mathrm{C}$ & Contrainte des institutions & Marginale & Majeure \\
\hline & Pouvoirs publics & Développement local & $\begin{array}{l}\text { Politique industrielle } \\
\text { et de l'innovation }\end{array}$ \\
\hline & $\begin{array}{l}\text { Grandes entreprises } \\
\text { Banques }\end{array}$ & $\begin{array}{l}\text { Veille et création } \\
\text { technologiques }\end{array}$ & $\begin{array}{l}\text { Couverture du marché } \\
\text { des PME }\end{array}$ \\
\hline $3^{\circ} \mathrm{I}$ & Innovation privée & $\begin{array}{l}\text { Majeure } \\
\text { OCR majoritairement } \\
\text { indépendants }\end{array}$ & $\begin{array}{l}\text { Mineure } \\
\text { Dans le cadre défini par } \\
\text { les pouvoirs publics }\end{array}$ \\
\hline $4^{\circ} \mathrm{I}$ & Innovation publique & $\begin{array}{l}\text { Mineure } \\
\text { OCR locaux } \\
\text { Programmes connexes } \\
\text { (SBIR, SBIC) }\end{array}$ & $\begin{array}{l}\text { Majeure } \\
\text { Statuts juridiques et fiscaux } \\
\text { Fonds d'assurance } \\
\text { Second marché }\end{array}$ \\
\hline & $\begin{array}{l}\text { Dialectique } \\
\text { réglementaire }\end{array}$ & $\begin{array}{l}\text { Non: } \\
\text { Réglementation et } \\
\text { fiscalités subies par les OCR }\end{array}$ & $\begin{array}{l}\text { Non: } \\
\text { Dynamique publique comme } \\
\text { facteur de dynamique privée }\end{array}$ \\
\hline
\end{tabular}

Tout d'abord, les OCR indépendants étaient à l'origine en 1989 d'environ $1 / 3$ du volume d'investissement en France contre près de $80 \%$ aux États-Unis. Les OCR affiliés à des banques ou institutions financières constituent le groupe désormais prépondérant en France, tandis qu'aux États-Unis, ces organismes sont généralement indépendants tout en recourant massivement à des capitaux collectés auprès d'institutions financières.

Ensuite, en partie à cause de l'affiliation des organismes d'intermédiation, le segment, moins risqué, des entreprises en développement est privilégié en France, alors que les OCR américains sont moins réticents à s'engager sur le segment des entreprises en création. De même, les investissements dans les secteurs de haute technologie sont minoritaires en France et nettement majoritaires aux États-Unis. 
Cette opposition est donc un reflet de la différenciation des processus innovateurs. En les extrapolant, on constate que les obstacles que rencontreront ces industries à l'amorce de la décennie 90 s'opposent également.

- Dans le cas américain, ils sont liés au processus d'innovation privé et décentralisé : il s'agira essentiellement de gommer certains comportements pervers (surenchères) suscités par l'afflux massif des capitaux institutionnels. Les facteurs de dysfonctionnement qui ont provoqué une phase dépressive en 1984-1985 étaient encore virtuels à la fin des années 80 .

- Dans le cas français, les obstacles sont symptomatiques du processus d'innovation institutionnel et centralisé. Le danger est celui d'une segmentation excessive de l'industrie entre un groupe (dominé par les OCR bancaires) privilégiant les risques mineurs en aval du cycle de vie des entreprises et un groupe (incorporant les opérateurs anglosaxons) qui resterait plus proche du modèle originel.

Caractéristiques des intervenants et des pratiques du capital-risque

\begin{tabular}{|c|c|c|}
\hline & Etats-Unis & France \\
\hline \multirow[t]{3}{*}{$\begin{array}{l}1^{\circ} \text { Apport de capitaux } \\
\text { aux OCR }\end{array}$} & $\begin{array}{l}\text { Majoritairement } \\
\text { institutionnel }\end{array}$ & $\begin{array}{l}\text { Majoritairement } \\
\text { institutionnel }\end{array}$ \\
\hline & Caisses de retraite & Banques \\
\hline & Assurances & Assurances \\
\hline $2^{\circ} \begin{array}{l}\text { Groupe d'OCR } \\
\text { dominant }\end{array}$ & Indépendants & $\begin{array}{l}\text { Captifs et semi-captifs } \\
\text { financiers }\end{array}$ \\
\hline \multirow[t]{2}{*}{$3^{\circ}$ Entreprises financées } & $\begin{array}{l}\text { Volumes } \\
\text { développement/création }=1,5\end{array}$ & $\begin{array}{l}\text { Volumes } \\
\text { développement/création }=4\end{array}$ \\
\hline & $\begin{array}{l}\text { Investissement à dominante } \\
\text { haute technologie }>60 \%\end{array}$ & $\begin{array}{l}\text { Investissement à dominante } \\
\text { haute technologie }<30 \%\end{array}$ \\
\hline \multirow[t]{2}{*}{$\begin{array}{l}4^{\circ} \text { Modalités de sorties } \\
\text { majeures }\end{array}$} & & $\begin{array}{l}\text { Entreprise industrielle } \\
\text { ou financière }\end{array}$ \\
\hline & $\begin{array}{l}\text { Entreprise industrielle } \\
\text { ou financière }\end{array}$ & Rétrocession à l'entrepreneur \\
\hline
\end{tabular}




\section{Conclusion}

En combinant le découpage « demande de caractéristiques/contraintes des institutions » et le découpage " innovation publique/innovation privée », nous avons relevé, dans les cas français et américain, des modalités innovatrices dissymétriques, de même que les pratiques des industries du capital-risque qu'elles ont engendrées.

Première conclusion : la comparaison semble indiquer que la diffusion du capital-risque américain ne se plie pas à une transposition homothétique, mais requiert l'engagement d'un processus innovateur spécifique.

Notamment, les autres expériences nationales confirment largement l'importance des politiques publiques pour l'adaptation du financement à risque :

- L'émergence d'une industrie du capital-risque en Grande-Bretagne n'a été permise que par une politique originale consacrée au financement des PME.

- De même, l'expérience néerlandaise (dont le capital-risque tient les premiers rangs européens) s'apparente à une intervention publique volontariste caractérisée par une offre de capitaux aux $\mathrm{OCR}^{13}$.

Aucune des économies occidentales ne semble se prêter à une acclimatation naturelle du capital-risque ou engendrer un innovation spontanée comme aux États-Unis. Sans généralement atteindre le degré de volontarisme français, la majorité des pays de l'OCDE présentent des politiques spécifiques d'adaptation d'obédience publique, souvent par le relais d'organismes parapublics. Dans les économies où ces efforts ne sont pas engagés (par inertie publique en RFA ${ }^{14}$ ou par inadéquation avec la culture industrielle au Japon), le développement des industries du capital-risque s'effectue à un rythme faible.

Deuxième conclusion : Si les industries française et américaine se distinguent par les conditions de leur formation, les offreurs de capitaux qui ont contribué à l'amorce des processus (pouvoirs publics en France et investisseurs individuels aux États-Unis) représentent désormais une fraction marginale de l'apport total de capital. De part et d'autre de l'Atlantique, les capitaux à risque trouvent leur source majeure auprès des institutions financières ${ }^{15}$ qui conditionnent désormais l'accès des industries nationales du capital-risque à la maturité.

13. Près de $20 \%$ de l'offre de capitaux est d'origine publique contre $5 \%$ en moyenne dans la CEE.

14. En 1989, 5,4\% des investissements, 3,8\% de la collecte et $3,7 \%$ du portefeuille européen.

15. Au sens large, y compris les caisses de retraite. Toutefois, alors que les OCR américains conservent majoritairement leur indépendance, les OCR filialisés à ces institutions constituent le groupe dominant en France. 


\section{Bibliographie}

ABRAHAM, J.P. (1987), «Innovation financière et croissance économique », Revue d'Économie Financière, $\mathrm{n}^{\circ} 2$, septembre.

Acs, Z.J. et D. AUDRETSCH (1988), «Innovation in large and small firms : an analysis », American Economic Review, vol 78, $\mathrm{n}^{\circ} 4$, septembre.

ADAM, M.C. et A. FARBER (1987), «Les caractéristiques de l'investissement novateur », Cahiers Economiques de Bruxelles, $\mathrm{n}^{\circ} 116$.

AgliftTa, M. (1987), «Structures économiques et innovations financières », Revue d'Économie Financière, $\mathrm{n}^{\circ} 2$, septembre.

BARRY, C. (1990), "The role of venture capital in the creation of public companies », Journal of Financial Economics, p. 447-471.

DE BoIssIEU, C. (1986), «Quelques réflexions sur l'analyse économique des innovations financières », Économie Appliquée, tome XXXIX, $\mathrm{n}^{\circ} 3$.

CHAN, Y.S. (1983), «On the positive role of financial intermediation in allocation of venture capital in a market with imperfect information ", The Journal of Finance, $\mathrm{n}^{\circ} 5$, décembre.

European Venture Capital Association (1990), Venture capital in Europe 1988, La Haie, Vuga Publishers.

Geofron, P. (1990), « La formation d'une innovation financière : le capital-risque. Une analyse comparative des modèles américain et français », thèse de doctorat, Université Paris XIII.

GeofrRon, P. (1987), « Capital-risque et financement de l'innovation technologique », Revue d'Economie Industrielle, $\mathrm{n}^{\circ} 42,4$ trimestre.

Geofrron, P. (1987), «L'état et la dynamique du marché européen du capital-risque », Revue d'Economie Financière, $n^{\circ} 3$, décembre.

Greenbaum, S.I. et C.F. HaYwood (1971), « Secular change in the financial services industry ", Journal of Money, Credit and Banking, mai.

HARDOUIN, J.C. (1973), « L'apparition de l'innovation financière. Contribution à l'étude de ses éléments explicatifs », thèse complémentaire, Université de Rennes.

Heggestad, A. et W. SHEPHERD (1982) «The banking industry », dans W. Adams (éd.) The Structure of American Industry, New York, McGraw-Hill.

HeERTJe, A. (1988), « Technical and financial innovation, » dans A. Heertje (éd.), Innovation, Technology and Finance, Londres, Basil Blackwell.

KANE, E.J. (1983), « Policy implications of structural changes dans financial markets », American Economic Review, mai.

LANCASTER, K.J. (1966), «A new approach to consumer theory », Journal of Political Economy, vol. 74, avril.

LELAND, H. et D. PyLE (1977), «Information asymmetries, financial structure, and financial intermediation », Journal of Finance, $\mathrm{n}^{\circ} 32$.

MOWERY, D. et N. RosenBERG (1979), « The influence of market demand upon innovation ", Research Policy, avril.

OCDE (1986), Le capital-risque : contexte, développement et action des pouvoirs publics, Paris.

Podolski, T.M. (1986), Financial Innovation and the Money Supply, Londres, Basil Blackwell. 
RaINelli, M. (1986), L'analyse économique et les PME, Colloque International de l'AIDE, 24 et 25 janvier 1985, paru dans les Actes du Colloque, Bruxelles, De Boeck-Wesmael.

SAHLMAN, W.A. (1990), «The structure and governance of venture-capital organizations ", Journal of Financial Economics, p. 473-521.

SCHUMPETER, J. (1983), Théorie de l'évolution économique, Paris, Dalloz.

Silber, W.L. (1975), (éd.) Financial Innovation, Lexington, D.C. Heath.

SILBER, W.L. et M. BEN HORIM (1977), «Financial innovation : a linear programming approach », Journal of Banking and Finance, $\mathrm{n}^{\circ} 1$. 


\section{Annexes}

Caractéristiques des processus nationaux d'innovation financière

\begin{tabular}{|c|c|c|}
\hline & Etats-Unis & France \\
\hline $\begin{array}{l}1^{\circ} \text { Demande de nouvelles } \\
\text { caractéristiques }\end{array}$ & $\begin{array}{l}\text { Gestion de la } \\
\text { rentabilité/liquidité } \\
\text { Augmentation des risques }\end{array}$ & Idem \\
\hline $\begin{array}{l}2^{\circ} \text { Contrainte des } \\
\text { institutions }\end{array}$ & $\begin{array}{l}\text { Contraintes réglementaires } \\
\text { des institutions financières } \\
\text { Concurrence née de la } \\
\text { déréglementation }\end{array}$ & $\begin{array}{l}\text { Besoins de financement } \\
\text { public }\end{array}$ \\
\hline $3^{\circ}$ Innovation privée & Majeure & Marginale \\
\hline $4^{\circ}$ Innovation publique & Marginale & Majeure \\
\hline $4^{\circ}$ Dialectique réglementaire & Oui & $\begin{array}{l}\text { Non } \\
\text { Degré de réglementation } \\
\text { inhibant }\end{array}$ \\
\hline
\end{tabular}

\section{Évolution en volume des ressources cumulées de l'industrie du capital-risque aux États-Unis (en millions de dollars)}

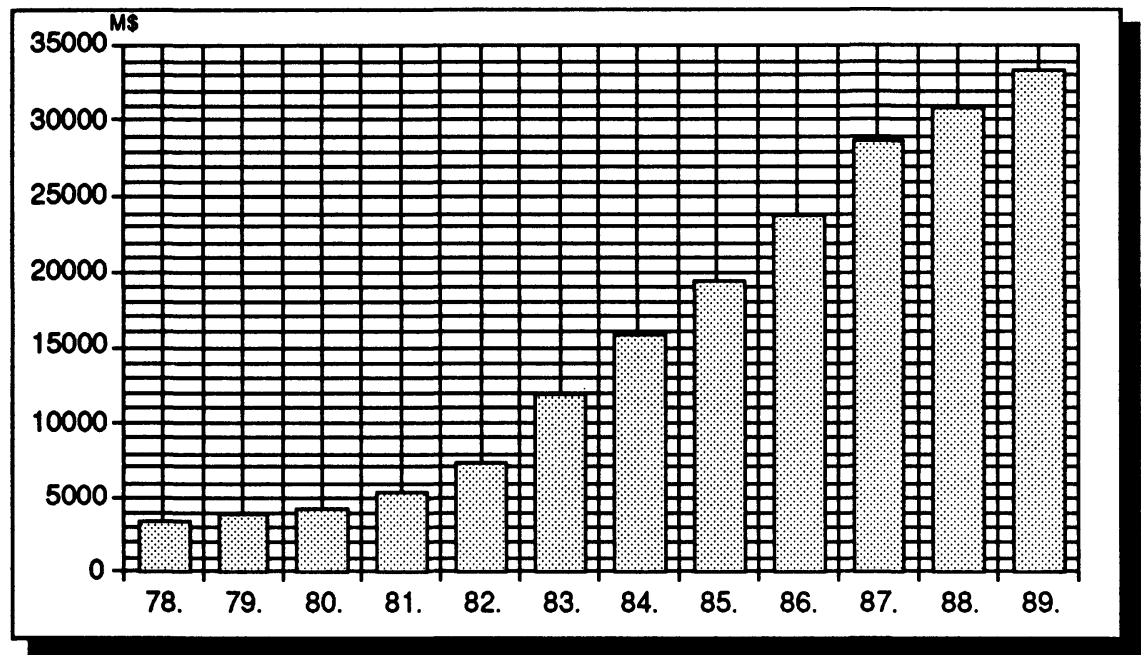


Flux de venture capital collectés et investis de 1970 à 1989 (en millions de dollars)

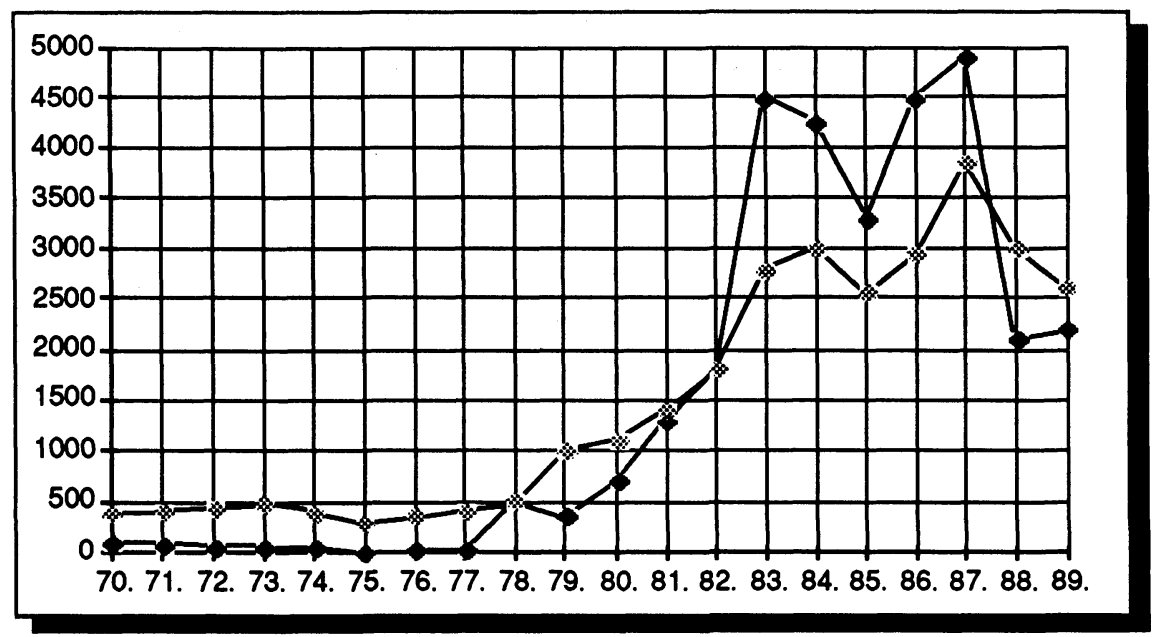

Investissements

- Collecte

Source : Venture Economics 1990. 